\title{
Night pain in arthritis: patients at risk from prescribed night sedation
}

\author{
P G Hardo, S A Wasti, A Tennant
}

\begin{abstract}
To assess the problem of night pain and the use of hypnotic drugs in patients with rheumatic diseases 165 consecutive patients (mean age 58.5 years) were assessed and questioned about night pain and the use of drugs including night sedation. Most of the patients (106 $(64 \%)$ ) were women. A total of $32(19 \%)$ patients were receiving night sedation for a mean duration of 43.9 months. Fourteen patients ( 13 women) were using these drugs to treat insomnia related to pain. The mean visual analogue pain score for night pain showed a significant difference between those receiving night sedation $(5 \cdot 2)$ and those who were not (3.7).

Of the 70 patients who answered the Stanford Health Assessment Questionnaire (HAQ), those receiving night sedation also had a significantly higher mean score (1.91) than those who were not (1.2), suggesting that patients receiving night sedation were more clinically disabled. Codeine was used by more (34\%) patients receiving night sedation than those who were not $(18 \%)$ suggesting that those receiving night sedation had more pain. These results highlight the need for better pain management in patients with rheumatic diseases to minimise the risk of prescribing addictive drugs such as hypnotic drugs and codeine.
\end{abstract}

(Ann Rheum Dis 1992; 51: 972-973)

Pain is the most common symptom of rheumatic disease. Most patients seek medical advice when the pain is disturbing and when it is not satisfactorily controlled it may lead to changes in personality, lifestyle, functional ability, depression, and sleep disturbance. ${ }^{1}$ The management of chronic pain in rheumatic diseases requires a multidisciplinary approach; however, relieving the suffering caused by pain in some patients remains difficult and is often inadequate. In one survey of patients with arthritis $29 \%$ were receiving benzodiazepines and most of these patients (70\%) used the drugs to overcome insomnia related to pain. ${ }^{2}$

Patients and methods

One hundred and sixty five patients with rheumatic disease admitted to hospital or attending a rheumatology outpatient clinic in the General Infirmary at Leeds were assessed and questioned about the use of drugs including hypnotic drugs. Their mean age was 58.5 years ( $95 \%$ confidence interval (CI) $49 \cdot 5$ to $67 \cdot 5$ ) and most (106) were women. Table l' shows that rheumatoid arthritis (RA) was the most common disease followed by osteoarthritis, ankylosing spondylitis, and non-articular rheumatism.

Night pain was assessed using a $10 \mathrm{~cm}$ visual analogue pain score varying from no pain to very severe pain. Individual joints, including the neck, upper and lower spine, shoulders, elbows, hands, hips, knees, ankles, and feet, were also assessed by applying a numerical pain scale of $0=$ no pain, $1=$ mild, $2=$ moderate, $3=$ severe, $4=$ unbearable. An overall pain score was created by summing these individual scores. Seventy patients also completed the Stanford Health Assessment Questionnaire (HAQ). It was thought that the use of a basic psychometric test such as the Hospital Anxiety and Depressions (HAD) Scale would not be useful for patients receiving tricyclic drugs or benzodiazepines.

\section{Results}

Table 1 shows that there was no significant difference in the number of patients with RA, osteoarthritis, or non-articular rheumatism receiving hypnotic drugs. A total of 32 patients (19\%) were receiving night sedation, $24(75 \%)$ of whom were women. The mean duration of night sedation was 43.9 months. Fourteen patients were receiving night sedation for insomnia related to pain, all but one of whom were women. There was no significant difference in the age of those receiving night sedation $(57 \cdot 3$ years) and those not $(63.2$ years) $(t=0.341)$.

Table 2 shows that about one third of patients receiving tricyclic drugs or codeine were also receiving night sedation. Although there was no significant difference in the use of non-steroidal anti-inflammatory drugs (NSAIDs) between those using night sedation and those not the analgesia produced by narcotics (codeine was usually taken in compound form with paracetamol such as co-codamol or co-dydramol) was used by significantly more (34\%) of those receiving night sedation than those not $(19 \%)$

Table 1 Diagnosis of patients with rheumatic diseases studied in this work

\begin{tabular}{lll}
\hline Dtagnosis & $\begin{array}{l}\text { Number of } \\
\text { patients }\end{array}$ & $\begin{array}{l}\text { No (\%) of } \\
\text { patients receiving } \\
\text { night sedation }\end{array}$ \\
\hline Rheumatoid arthritis & 53 & $14(26)$ \\
Osteoarthritis & 39 & $9(23)$ \\
Ankylosing spondylitis & 32 & $3(9)$ \\
Non-articular rheumatism & 18 & $5(28)$ \\
Other rheumatic diseases & 23 & $1(4)$ \\
Total & 165 & $32(19)$ \\
\hline
\end{tabular}


Table 2 Summary of the consumption of drugs by patients in this study

\begin{tabular}{lll}
\hline Drug & $\begin{array}{l}\text { Total number } \\
\text { of patients } \\
\text { receiving } \\
\text { other drugs }\end{array}$ & $\begin{array}{l}\text { No (\%) of } \\
\text { patients receiving } \\
\text { other drugs } \\
\text { also receiving } \\
\text { night sedation }\end{array}$ \\
\hline $\begin{array}{ll}\text { NSAIDs* } \\
\text { Tricyclic drugs }\end{array}$ & 95 & $19(20)$ \\
Second line treatment & 19 & $7(37)$ \\
Steroids & 43 & $10(23)$ \\
Narcotics & 24 & $6(25)$ \\
Codeine & & \\
Dextropropoxyphene + & 29 & $11(32)$ \\
paracetamol (co-proxamol) & $6(21)$ \\
\hline
\end{tabular}

${ }^{*}$ NSAIDs=non-steroidal anti-inflammatory drugs.

Table 3 Summary of the consumption of drugs by patients receiving night sedation and those not receiving night sedation. Results given as No of patients (\%)

\begin{tabular}{|c|c|c|}
\hline Drug & $\begin{array}{l}\text { Receiving night } \\
\text { sedation }\end{array}$ & $\begin{array}{l}\text { Not receiving } \\
\text { night sedation }\end{array}$ \\
\hline $\begin{array}{l}\text { NSAIDs* } \\
\text { Tricyclics } \\
\text { Secónd line treatment } \\
\text { Steroids }\end{array}$ & $\begin{aligned} & 19(59) \\
& 7(22) \\
& 10(31) \\
& 6(19)\end{aligned}$ & $\begin{array}{l}75(56) \\
12(9) \\
33(25) \\
18(14)\end{array}$ \\
\hline $\begin{array}{l}\text { Narcotics } \\
\text { Codeine } \\
\text { Dextropropoxyphene }+ \\
\text { paracetamol (co-proxamol) }\end{array}$ & $\begin{array}{r}11(34) \\
6(19)\end{array}$ & $\begin{array}{l}24(18) \\
23(17)\end{array}$ \\
\hline Total No of patients & 32 & 133 \\
\hline
\end{tabular}

"NSAIDs=non-steroidal anti-inflammatory drugs.

$\left(\chi^{2} 3.93 ; p=<0.05\right)$ (table 3). This suggests that those patients receiving night sedation had more pain which was not controlled by NSAIDs alone.

This is supported by the mean visual analogue pain score which shows a significant difference between those receiving night sedation $(5 \cdot 3)$ and those not (3.7) $(t=2 \cdot 77 ; p<0 \cdot 01)$. Likewise the total pain score was significantly higher among those receiving night sedation (13.3) than those not $(8 \cdot 6)(t=2 \cdot 26 ; p<0 \cdot 05)$. Of the 70 patients who answered the HAQ, the 15 receiving night sedation were also found to have a significantly higher mean score (1.91) compared with those not receiving night sedation $(1 \cdot 2)(t=2.67 ; p<0.01)$ which suggests that in addition to experiencing more pain, patients using night sedation were also more clinically disabled.

Although the results did not reach statistical significance patients receiving night sedation were more likely to be living alone $(30 \%)$ than those not $(14 \%)$ and this may indicate an association between awareness of night pain and social isolation.

\section{Discussion}

The results of this study indicate that the patients with rheumatic diseases who appear to be at increased risk of taking night sedation are those who live alone, suffer more pain, are more disabled, and are likely to be prescribed codeine analgesia or tricyclic drugs. It is not clear why more women used night sedation for insomnia associated with pain. This may be due to differences in pain threshold or anxiety levels and warrants further investigation.

It was interesting that a similar proportion of patients with RA and non-articular rheumatism were receiving night sedation (table 1 ), emphasising the disability experienced by the latter group. Why such a small proportion of patients with ankylosing spondylitis (9\%) were receiving night sedation also needs explanation. It is possible that the level of anxiety and depression experienced by patients may explain the reason for one third of patients receiving tricyclic drugs also taking night sedation (table 2). It was difficult, however, to determine whether tricyclic drug treatment was primarily prescribed for reactive depression or for central analgesia effect because most patients had been receiving these drugs over a long period of time.

Night pain and sleep disturbance in patients with arthritis require full attention and a multidisciplinary team management approach. Effective communication is essential between the patient and the team. Any evidence of anxiety or depression through the course of the disease should first be tackled with counselling. As night sedation is often prescribed for insomnia associated with pain, modification of the NSAID/analgesic regimen should be tried first. Nefopam (Acupan) is often a useful analgesic and has the advantage of not causing habituation. ${ }^{3}$ One of us (PH) has found it useful in combination with NSAIDs.

The unnecessary prescribing of benzodiazepine carries the risk of dependency with knock-on effects in lost working capacity due to side effects and their treatment. ${ }^{4}$ The number of patients seeking damages for the symptoms of addiction and of withdrawal from these drugs is growing. ${ }^{5}$ In our study the duration of night sedation varied from six weeks up to 14 years (mean 43.9 months) which indicates that many patients were becoming addicted to these drugs. Although a plan to withdraw hypnotic drug treatment was not part of the current project, elsewhere the introduction of such a policy to withdraw psychotropic drugs with the support of a specially trained practice nurse has shown some success. ${ }^{6}$

Developing a drug audit in patients with rheumatic diseases appears to be a useful and informative first step in highlighting these problems.

We thank Professor Verna Wright for his helpful comments on an earlier version of this paper.

1 Pavne R, Max M, Inturrisi C, et al. Principle of analgesic use in treatment of acute pain or chronic cancer pain. Clin Pharm 1987; 6: 523-32.

2 Hardo P G, Kennedy T D. Night sedation and arthritic pain J $R$ Soc Med 1991; 84: 73-5.

3 ABPI Data Sheet Compendium. London: Datapharm Publication Limited, 1990: 959.

4 Brahms D. Benzodiazepine overprescribing: successful initiative in New York State. Lancet 1990; i: 1372-3.

5 Dyer C. Benzodiazepine claims. BMF 1991; 303: 79.

6 Jones $\mathrm{D}$. Weaning elderly patients off psychotropic drugs in general practice: a randomised control trial. Health Trends 1991; 22: 164-6. 